\title{
Eshelby twist and correlation effects in diffraction from nanocrystals
}

\author{
A. Leonardi, ${ }^{1}$ S. Ryu, ${ }^{2}$ N. M. Pugno, ${ }^{3,4,5}$ and P. Scardi ${ }^{1, a)}$ \\ ${ }^{1}$ Department of Civil, Environmental and Mechanical Engineering, University of Trento, Trento 38123, Italy \\ ${ }^{2}$ Department of Mechanical Engineering, Korea Advanced Institute of Science and Technology (KAIST), \\ Daejeon 305-338, Korea \\ ${ }^{3}$ Department of Civil, Environmental and Mechanical Engineering, Laboratory of Bio-Inspired \\ and Graphene Nanomechanics, Universita' di Trento, via Mesiano, 77, 38123 Trento, Italy \\ ${ }^{4}$ Center for Materials and Microsystems, Fondazione Bruno Kessler, Via Sommarive 18, 38123 Povo (Trento), \\ Italy \\ ${ }^{5}$ School of Engineering and Materials Science, Queen Mary University of London, Mile End Road, \\ London E1 4NS, United Kingdom
}

(Received 7 January 2015; accepted 13 April 2015; published online 24 April 2015)

\begin{abstract}
Molecular dynamics simulations were used to model the Eshelby dislocation inside Pd and Ir nanowires and to predict the powder diffraction pattern using the Debye scattering equation. We find that the ideal dislocation solution by Eshelby is in good agreement with the observed twist angle and deviatoric strain, even though it ignores both the splitting of the Eshelby dislocation into two partials and surface stress. Surface stress plays a significant role only for nanorods with small aspect ratio $(\sim 1: 1)$. We also find that Wilson's prediction on the diffraction peak broadening for the Eshelby dislocation is overestimated because it ignores the fact that the Eshelby twist relaxes the deviatoric strain. Moreover, the twist loosens the correlation along the nanorod, causing additional line profile broadening, which is read by diffraction as a decrease of coherent domain size when the total twist angle is bigger than $1.5^{\circ}$. Overall, our findings suggest a novel way to predict and analyze the dislocations as well as the resulting strain fields in the twisted nanocrystalline rods. (C) 2015 AIP Publishing LLC. [http://dx.doi.org/10.1063/1.4918918]
\end{abstract}

\section{INTRODUCTION}

Eshelby showed, in $1953,{ }^{1}$ that screw dislocations parallel to the axis of thin rods are stable if they lie within a central region, where image forces cannot draw them out. However, edge dislocations are unstable and tend to slip away from the crystal lattice. The elastic field and stability of single or multiple Eshelby dislocations can be calculated using more details, e.g., by taking into account the mixed dislocation type, the split into partials in face-centered-cubic (FCC) lattices, and surface stress. However, as shown in this paper, the main result from Eshelby's model, the twisting of a thin rod in proportion to the total screw component of dislocations, is valid except in the extreme case of thin disks, i.e., large diameter compared to height.

The so-called Eshelby twist is a consequence of the mechanical equilibrium met by a screw dislocation in a cylindrical rod (with at least one free end). The dislocation-induced torque twists the elastic medium by an angle $\alpha=-b /\left[\pi R^{2}\right]$ (twist angle per unit length), ${ }^{1}$ where $b$ is the modulus of the Burgers vector and $R$ is the rod radius. In a rod of length $H$, the total twist is simply obtained as $\alpha H$. As a consequence the twist angle is small and often hard to detect experimentally, except in needle-like crystalline domains with high aspect ratios.

Recent work on nanowires and similar systems ${ }^{2,3}$ has drawn attention to the possibility of measuring Eshelby twist and exploiting its effect practically. ${ }^{4}$ The discovery of

\footnotetext{
a) Author to whom correspondence should be addressed. Electronic mail: Paolo.Scardi@unitn.it.
}

branched nanowires and the chiral pattern produced by the Eshelby twist allows for the observation of this twist using real space imaging tools such as scanning electron microscopes. An alternative technique for the observation of dislocations and twist, which Eshelby mentioned in his original paper, is X-ray diffraction (XRD). Very soon ${ }^{1}$ after Eshelby's original paper AJC Wilson ${ }^{5}$ tackled the problem of determining the diffraction line broadening effect of a straight dislocation in a cylindrical rod, providing approximate expressions for integral breadths. The underlying hypothesis, however, only considered the displacement component along the screw dislocation line, $u_{z}=b \theta /[2 \pi]$, disregarding any twist or rotational components that surely affect the position of the scattering centers.

This might appear as a minor problem, limited to the evolution of the theory of dislocations and diffraction. However, all following theoretical developments, like those made by Krivolglaz ${ }^{6-9}$ and by Wilkens, ${ }^{10,11}$ were based on the same assumption. This approximation, which is a useful simplification, may be acceptable in a few limited cases, e.g., for geometrically constrained crystalline domains (as in a polycrystalline microstructure), but it might be quite misleading for free crystalline domains. The question arises whether the Eshelby twist can influence the correlation between scattering centers that is the fundamental mechanism underlying diffraction from crystalline media. In the present work, using FCC crystalline (Pd and Ir) nanorods as examples, this fundamental problem is tackled by using two different approaches: continuum mechanics with some details added to the original Eshelby solution, and an atomistic approach, based on Molecular Dynamics (MD) simulations. 


\section{ESHELBY TWIST BY TWO SHOCKLEY PARTIAL DISLOCATIONS}

The original Eshelby's paper in 1953 discusses the perfect screw dislocation lying along the axis of thin rods. ${ }^{1}$ However, in FCC crystals, the perfect screw dislocation with the Burgers vector $\vec{b}=\frac{a}{2}\langle 110\rangle$ tends to dissociate into two partial dislocations having Burgers vectors $\vec{b}_{p}=\frac{a}{6}\langle 121\rangle$ and $\vec{b}_{p}=\frac{a}{6}\langle 21 \overline{1}\rangle$, because this reaction lowers the total energy of the system. ${ }^{12}$ As shown in more detail below, the actual distance between two partials is limited by the formation of a stacking fault ribbon with width $d$ (see Fig. 1): the larger the faulting energy, the smaller the separation between partials.

The screw components with $\left|\overrightarrow{b_{s}}\right|=\frac{2\left|\overrightarrow{b_{p}}\right|}{\sqrt{3}}$ induce the repulsive elastic force $\mu b_{s}^{2} /[2 \pi d]$ per unit length, while antiparallel edge components with $\left|\overrightarrow{b_{e}}\right|=\frac{\left|\overrightarrow{b_{p}}\right|}{\sqrt{3}}$ generate the attractive elastic force $\mu b_{e}^{2} /[2 \pi(1-\nu) d]$ per unit length between two partial dislocations, where $\mu$ and $\nu$ are the effective isotropic shear modulus and Poisson ratio, respectively. As the screw components are larger, the overall elastic interaction between dislocation pairs is repulsive. In addition, a stacking fault with energy $\gamma$ generates an additional attractive force (equal to $\gamma$ per unit length). The balance between repulsive and attractive forces dictates the equilibrium distance $d_{e}=\frac{\mu}{2 \pi \gamma}\left(b_{s}^{2}-\frac{b_{e}^{2}}{1-\nu}\right)$. Cubic elastic constants, stacking fault energy, and lattice constants of Pd were computed, using the Embedded Atom Model (EAM) potential (see below), to be, $\mathrm{C}_{11}=235 \mathrm{GPa}, \mathrm{C}_{12}=180 \mathrm{GPa}, \mathrm{C}_{44}=82 \mathrm{GPa}, \gamma$ $=169 \mathrm{~mJ} / \mathrm{m}^{3}, \mathrm{a}_{0}=0.3890 \mathrm{~nm} .{ }^{13}$ respectively. Following the approach of Scattergood and Bacon for the anisotropic elastic medium, ${ }^{14,15}$ we obtain an effective shear modulus $\mu=47.5 \mathrm{GPa}$ and a Poisson ratio $\nu=0.466$. This leads to $d_{e}=0.318 \mathrm{~nm}$. Such a short distance means the separation between partials is always much smaller than the diameter of the nanorod, allowing us to disregard image forces acting on the dislocation from the free surface of the nanorod.

Because the edge component does not induce any torque, only screw dislocation components contribute to the twist angle of a nanowire. When a screw dislocation is

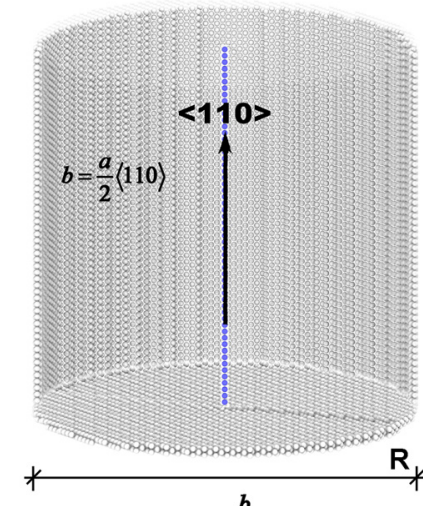

(a)

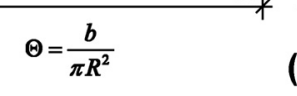

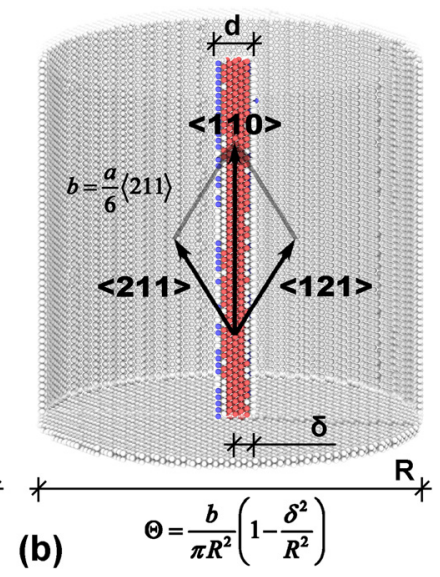

FIG. 1. Dissociation of an ideal screw dislocation (a) into two Shockley partial dislocations with separation distance $d(b)$. Stacking fault is generated between the two partial dislocations after dissociation. located at distance $\delta$ from the center, the Eshelby twist angle per unit length is given by $\frac{b_{s}}{\pi R^{2}}\left(1-\frac{\delta^{2}}{R^{2}}\right)$ as shown by Weinberger in 2011. ${ }^{16}$ Summing the twist angles from two partial dislocations, we obtain $\frac{2 b_{s}}{\pi R^{2}}\left(1-\frac{\delta^{2}}{R^{2}}\right)=\frac{b}{\pi R^{2}}\left(1-\frac{\delta^{2}}{R^{2}}\right)$. Because the correction term $\frac{\delta^{2}}{R^{2}}$ is less than $0.04 \%$ when $R$ is $8 \mathrm{~nm}$, we can compute the Eshelby twist angle neglecting the contribution of the Shockley partial dissociation.

\section{MD SIMULATION OF CYLINDRICAL ROD NANOCRYSTALS WITH AXIAL SCREW DISLOCATION}

We study cylindrical Pd nanocrystals of diameter (D) and height $(\mathrm{H})$ ranging from 8 to $64 \mathrm{~nm}$, with and without screw dislocations (described by a shift of the atomic coordinates as $\left.u_{z}=b \theta /[2 \pi]\right)^{17}$ along the [hh0] axis, with the FCC unit cell oriented as shown in Fig. 2. The MD simulations were performed via LAMMPS code (Large-scale Atomic/ Molecular Massively Parallel Simulator ${ }^{18}$ employing the Embedded Atom Method potential ${ }^{19-21}$ and Langevin thermostat at $300 \mathrm{~K}$ combined with a constant NPT (constant Pressure - $0 \mathrm{~Pa}$ - and constant Temperature) integration with $1 \mathrm{fs}$ time step. Next to an equilibration period of $1 \mathrm{~ns}$, a time trajectory of the atomic positions in space was recorded as a sequence of 100 independent microstructure snapshots sampled at a time interval of 1 ps. Finally, the space arrangement of the atom coordinates was averaged over the time trajectory so to cancel out any dynamic contribution to the distortion field.

As clearly visible from a top view (Fig. 2(d)), the screw dislocation line with Burgers vector $\vec{b}=\frac{a}{2}\langle 110\rangle$ splits in two partials with $\vec{b}_{p}=\frac{a}{6}\langle 121\rangle$ and $\vec{b}_{p}=\frac{a}{6}\langle 21 \overline{1}\rangle$. The picture also shows the twist resulting from the dislocation shear strain discussed in Sec. II. The dislocation line and the partials also seem to be twisted, as shown in Fig. 2(e). This is because upper and lower parts of the dislocation line split into two different $\{111\}$ slip planes with a relative angle of $70.5^{\circ}$. This configuration is energetically favorable compared to that in which a dislocation splits into one $\{111\}$ slip plane, because per unit length screw dislocations have lower energy than the edge dislocations and thus the burgers vectors and dislocation lines become more parallel.

MD simulations show not only the morphology of the screw dislocation but also the strain field to be different from the ideal screw dislocation solution of continuum mechanics. Fig. 3 shows that the dislocation line is split into two partials (cf. Fig. 2) this introduces a narrow region of faulted atomic layer stacking. The two partial dislocation lines are positioned asymmetrically due to the surface step at the top and bottom surface of the nanorods. Things are further complicated by the surface relaxation effect, caused by under-coordination of the $\mathrm{Pd}$ surface atoms. Despite all the complexities, the twist angle provided by MD simulations closely matches the one derived from the Eshelby formula of continuum mechanics (Figs. 4(a) and 4(b)).

All this is shown in detail for both Pd and Ir cylinders with fixed height and increasing diameter. Similar evidence 

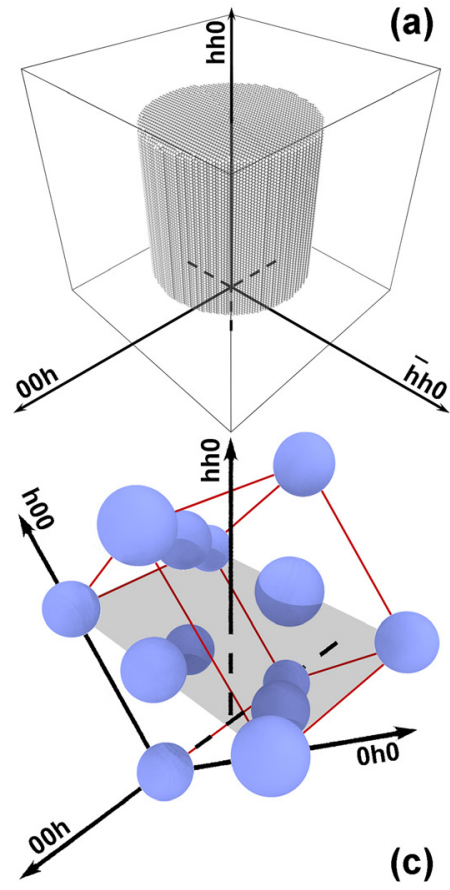

(c) (a)

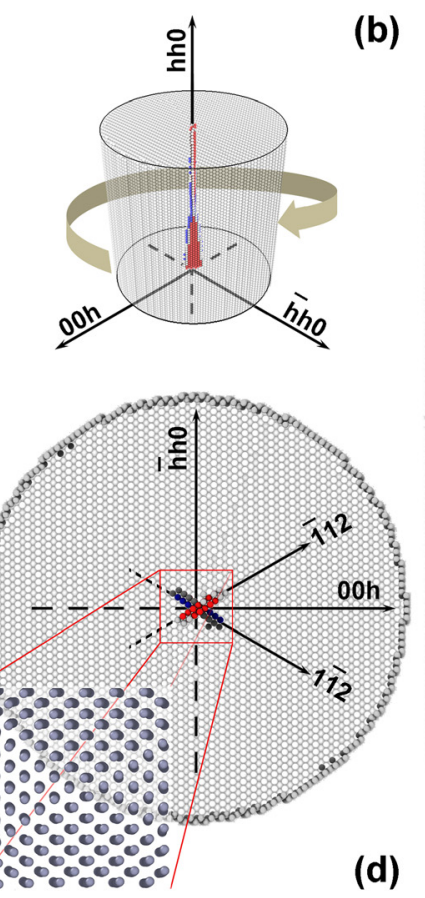

(b)

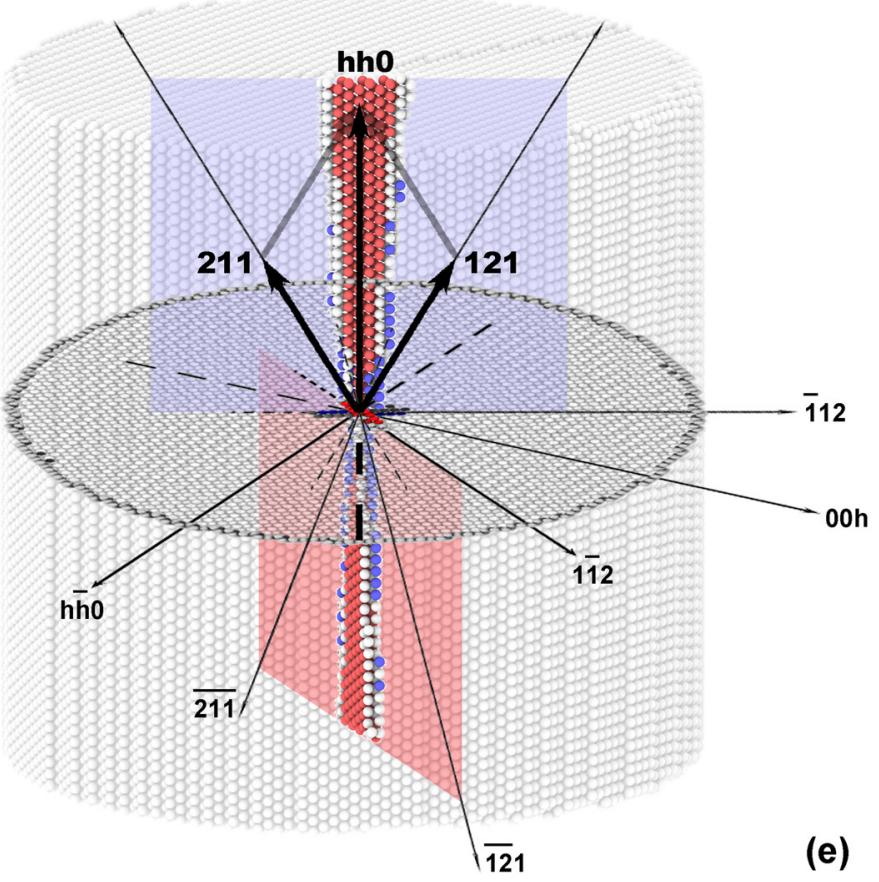

FIG. 2. MD simulation of cylindrical Pd nanocrystals without (a) and with (b) a screw dislocation laying on the [hh0] axis. Crystallographic orientation is schematically shown in (c), whereas the top view in (d) shows details of the dislocation split in partials and the twist, which is also shown in more detail in (e).

emerged from simulations made in a temperature range of $200 \mathrm{~K}$ to $600 \mathrm{~K}$. The calculated twist rate closely agrees with values given by the Eshelby formula, which is little affected by the split in partials of the screw dislocation. Significant deviations are only observed for disk-shaped nanocrystals, i.e., for high $\mathrm{D} / \mathrm{H}$ ratios, where the twist angle from MD simulations is markedly smaller than the value from the continuum mechanics models of the previous section. Such deviations (Figs. 4(c) and 4(d)) are caused by the boundary condition imposed by the free surface which becomes increasingly relevant for growing $\mathrm{D} / \mathrm{H}$ ratio.

Deviations are also observed for the opposite condition, i.e., very low $\mathrm{D} / \mathrm{H}$ ratios, although surface effects seem to be much less relevant for needle-like nanocrystals. Further details are provided by simulations with a variable step on the top-bottom surface: it was found that, by increasing TopBottom step on the surface to $b$, the twist angle increases, still remaining below the expected Eshelby value.
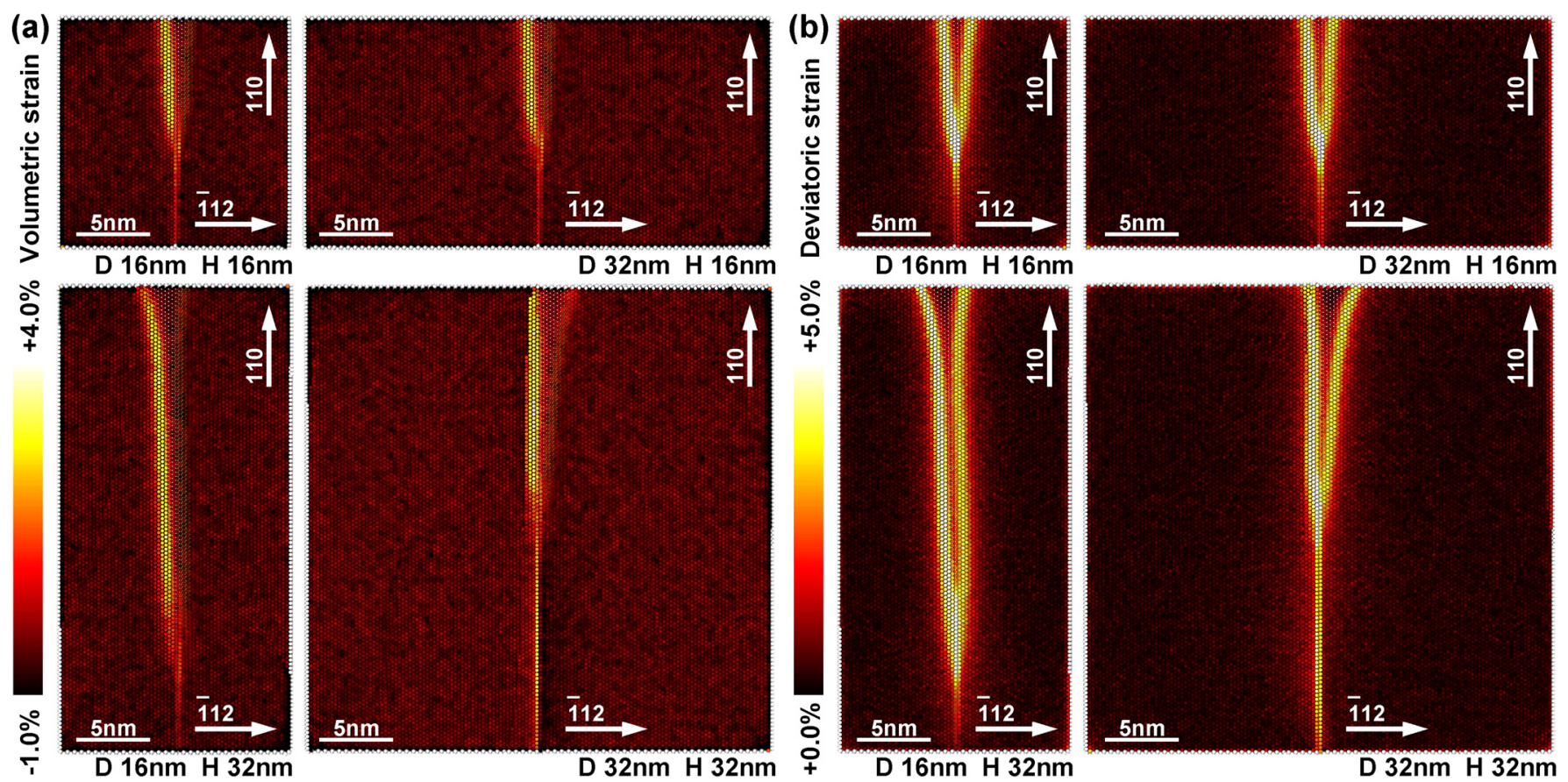

FIG. 3. Isotropic (volumetric) (a) and deviatoric (b) strain on [1 12]/[110] cross sections of MD simulated cylinders with axial screw dislocation for different diameter (D) and height (H). 

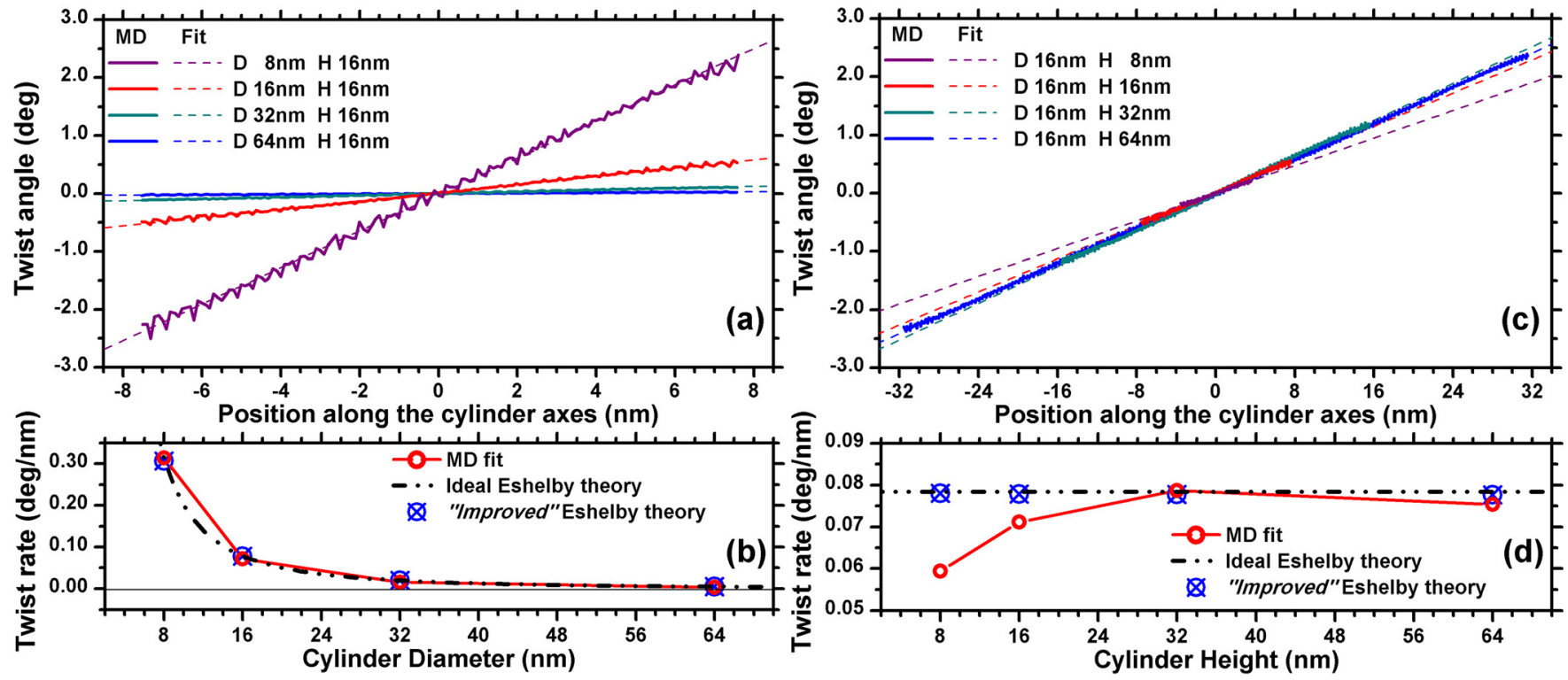

FIG. 4. Eshelby twist angle (a), (c) and rate (b), (d) for MD simulated cylindrical Pd nanocrystals with fixed height (H) and variable diameter (D) (left), and vice-versa (right). Ideal Eshelby theory refers to a straight line whole screw dislocation, whereas the improved model is from the present paper.

Free surfaces have a marked effect even in the absence of any line defect. Because of the surface strain field, the average FCC unit cell of Pd in the MD simulation undergoes a monoclinic distortion. As shown in Fig. 5 for the cylindrical domain of Fig. 4, the main effect is that the [h00] cube edge perpendicular to the [hh0] cylinder axis (cf. Fig. 1) is generally different from the other two ([h00] and [0h0]) edges. The trend changes with the cylinder aspect ratio $(\mathrm{D} / \mathrm{H})$, as an effect of the correspondingly different ratio between lateral surface and top-bottom surfaces. The presence of the axial screw dislocation does not qualitatively change the picture, but amplifies the distortion, especially for needle-like cylinders (larger lateral surface). It is worth noting the distortion tends to disappear for the $\mathrm{D}=\mathrm{H}$ case, which makes the cylinder shape closer to the symmetry requirement of the cubic elastic tensor.

As expected, the main strain component is deviatoric. Fig. 6 shows trends of deviatoric strain as a function of the radial distance from the cylinder axis. MD results refer to the cylindrical domains of Fig. 5, at half cylinder height ((a), (c)
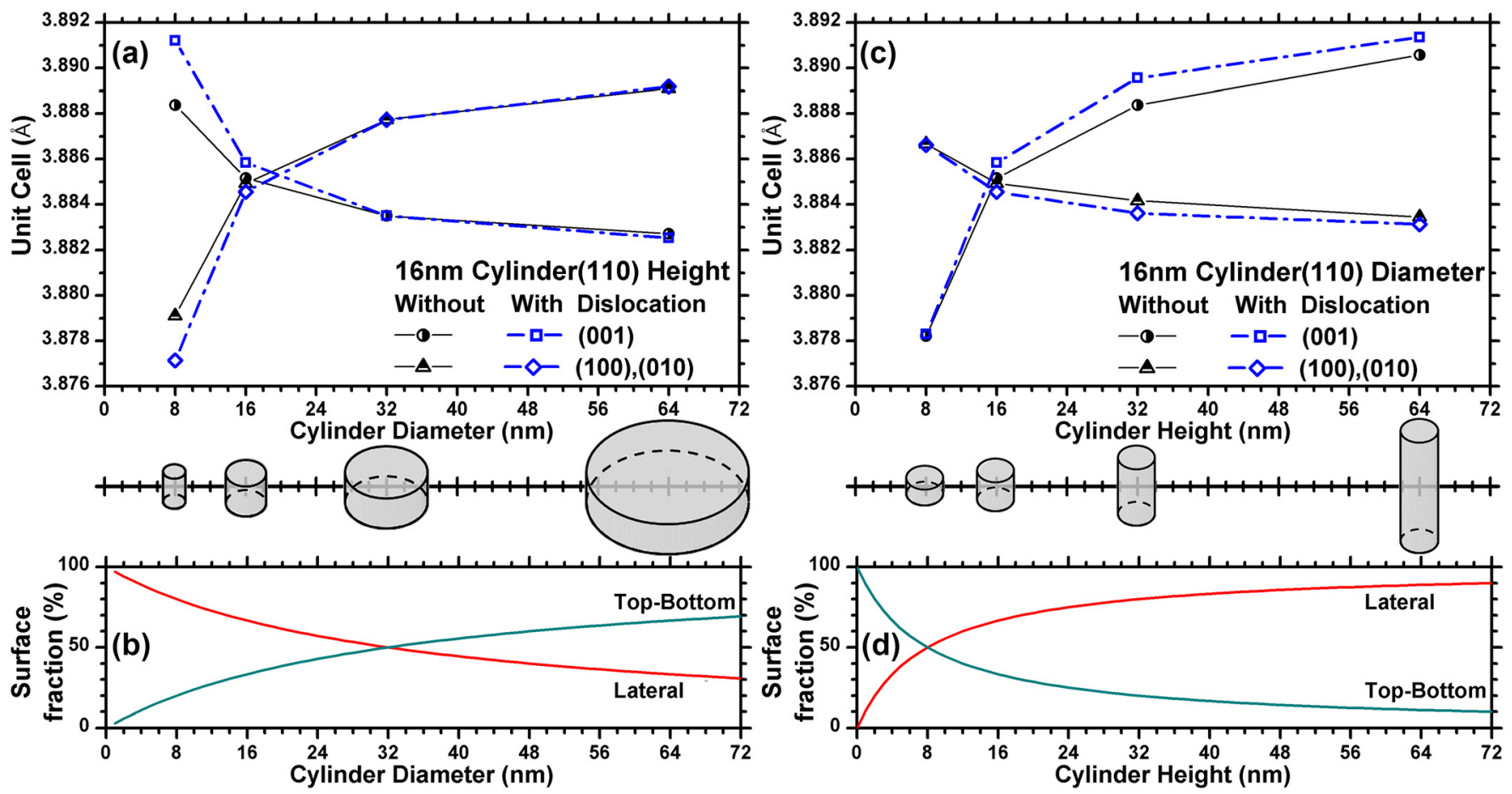

FIG. 5. Unit cell parameters (FCC Pd cube edge, refer to Fig. 1(c)) from MD simulated cylinders with different D/H aspect ratios: with fixed (16nm) height and varying diameter (a) and vice versa (c). Trends are shown with (dashed) and without (line) screw dislocation laying on the [hh0] axis. Corresponding fractions of lateral surface and top-bottom surface are shown below (b), (d). 

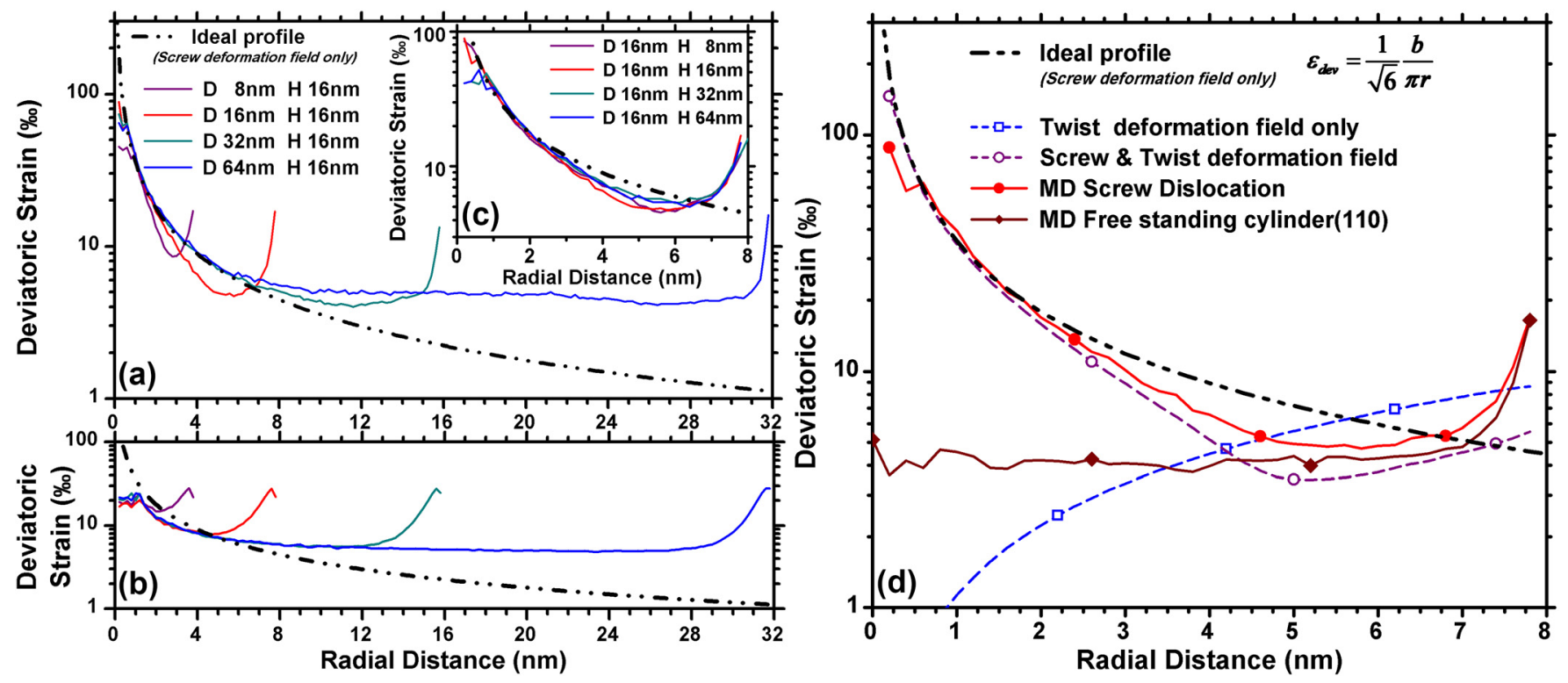

FIG. 6. Deviatoric strain as a function of the radial distance for MD simulated nanocrystals with screw dislocation along the [hh0] axis. On the left (a), trend observed for $\mathrm{H}=16 \mathrm{~nm}$ and different diameters on the central cross-section, and near the cylinder basis (b). The inset (c) shows the case of constant diameter $(\mathrm{D}=16 \mathrm{~nm})$ and varying height. On the right $(\mathrm{d})$, detail of results for the $\mathrm{D}=\mathrm{H}=16 \mathrm{~nm}$ cylinder with axial screw dislocation: see text for details.

and (d)) and near the cylinder bases (b), respectively. The trend according to the continuum mechanics expression for an ideal screw dislocation (dash-dot line) in an infinite medium diverges in the core region as $\varepsilon_{d e v}=\frac{1}{\sqrt{6}} \frac{b}{\pi r}$ (obtained as the deviatoric component ${ }^{22}$ using the standard equation for the strain tensor ${ }^{17}$ ), whereas MD values are high but finite, forming a plateau across a finite thickness region around the partial dislocations.

The infinite medium continuum mechanics expression is only matched over a short range just outside the core region. Moving in a radial direction toward the free surface, deviatoric strain from MD simulations stops decreasing and starts increasing again, owing to the surface relaxation effect. MD simulations confirm that cylinder height has no relevance on the radial distribution of the deviatoric strain (Fig. 6(c)), whereas near the free basis surfaces of the cylinder (b) strain trend is flatter, mostly related to the surface relaxation effect.

Results for the $\mathrm{D}=\mathrm{H}=16 \mathrm{~nm}$ cylinder are shown again in Fig. 6(d). As discussed previously, this is the case where the cylindrical shape is closer to the elastic symmetry restrictions of a cubic phase. The MD trend for the same cylinder without dislocation, where the deviatoric strain is solely given by the surface relaxation effect is also shown. Similarity with trends in Fig. 6(b) is quite evident. These results explain why there is a marked deviation from the infinite medium continuum mechanics expression. However, such deviations are diminished by the Eshelby twist, which is responsible for a deviatoric strain component that increases with the radial distance, so that the combined effect (screw and twist) gets closer to the MD simulation.

\section{DIFFRACTION FROM FREE NANOCRYSTALLINE RODS WITH AXIAL DISLOCATIONS}

From the previous discussion, it is quite evident that, even in the best case ( $H=D$ cylinder), the twist tends to reduce the shear strain considerably with respect to the infinite medium continuum mechanics prediction. In the following we discuss how the twist-and more in general, the complex morphology of dislocations in a free nanocrystalinfluences the scattering of radiation.

Given the atomic coordinates from MD simulations, it is a simple task to use the Debye Scattering Equation $(\mathrm{DSE})^{23-25}$ to calculate the diffraction pattern for a powder of cylindrical domains, i.e., a powder or a polycrystalline aggregate made of identical domains with random orientation. In Fig. 7, the simulated patterns for a powder of $\mathrm{D}=\mathrm{H}$ $=16 \mathrm{~nm}$ cylinders is shown for perfect (crystallographic)
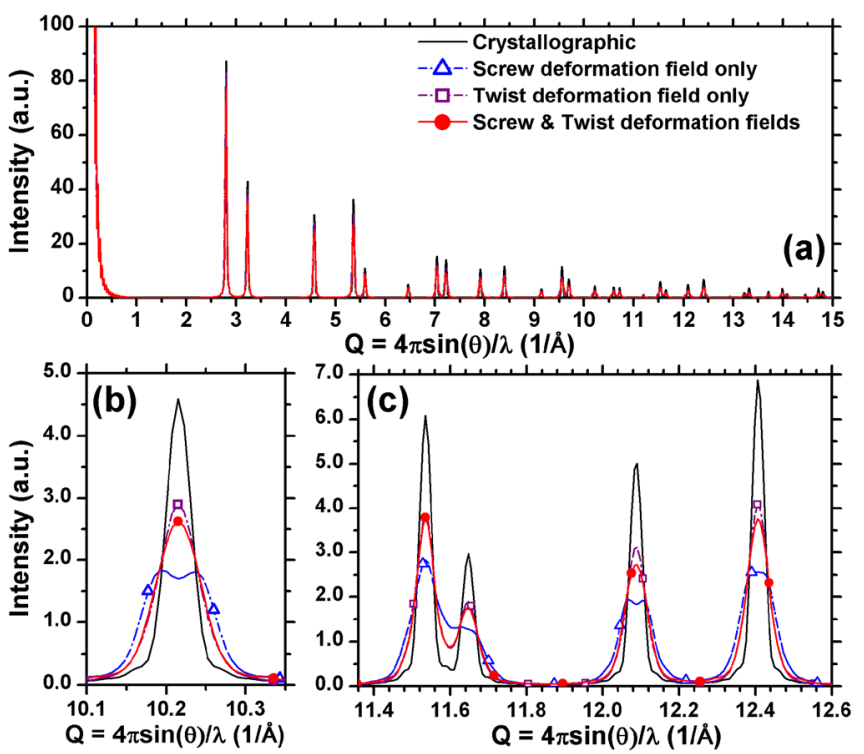

FIG. 7. Powder Diffraction patterns of Pd cylindrical nanocrystals without and with screw dislocations, given as scattered intensity as a function of Q, the wavevector transfer modulus (a). Details are highlighted in (b) and (c). Patterns are obtained by applying the Debye Scattering Equation to atomic coordinates provided by MD simulations (see text for details). 
crystals and after the application of atomic displacement due to screw, twist, and screw plus twist. Here, screw is referred to the strain field from an ideal screw dislocation in an infinite medium. It is interesting to note that the twist decreases the broadening of the diffraction peak profiles, which is an indication of a reduced strain effect with respect to the screw-only case. Screw is responsible for the split of some diffraction lines, most notably the (hh0) lines, as a direct effect of the shear strain of the screw dislocation laying on the [hh0] axis. This feature is smoothed and disappears when the twist effect is combined with the shear strain (screw + twist). Further interesting features can be observed by comparing powder patterns and corresponding singlecrystal reciprocal space (RS) intensity distributions. This is shown for Pd cylinders of different diameter and height (Fig. 8) all with a screw dislocation along the [hh0] axis.

Cylinders with smaller diameters ((a), (b), and (c)) have broader peak profiles, the broadening being more visible for increasing Q (b), as the dislocation density is proportionally larger in a needle-like (low $\mathrm{D} / \mathrm{H})$ cylinder than in a flat disk. Domain shape and strain anisotropy combine to give different broadening of the diffraction maxima, a feature most

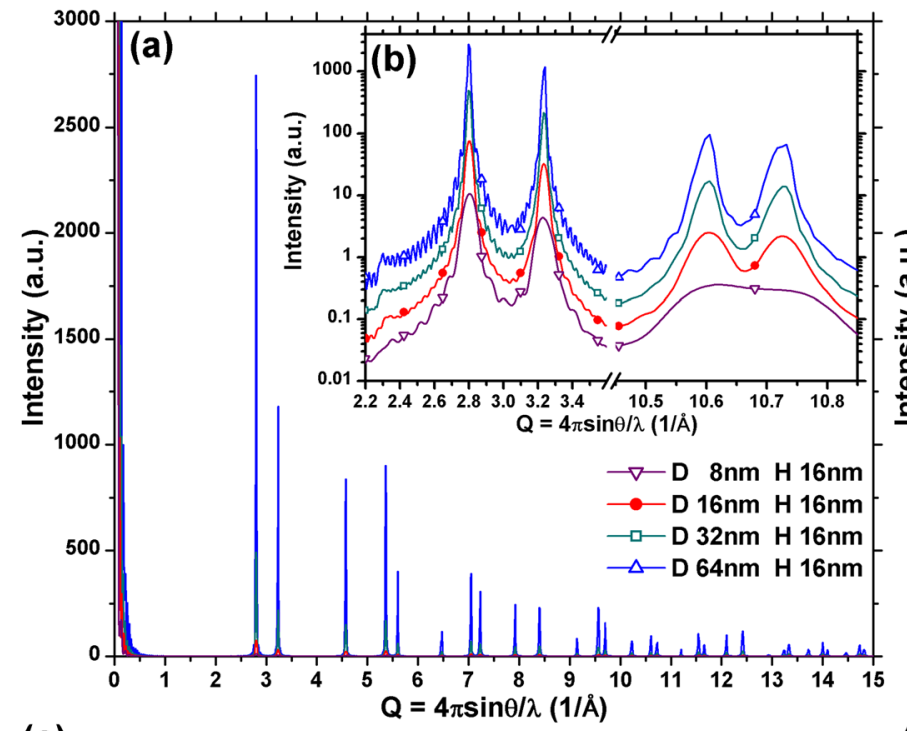

(c)

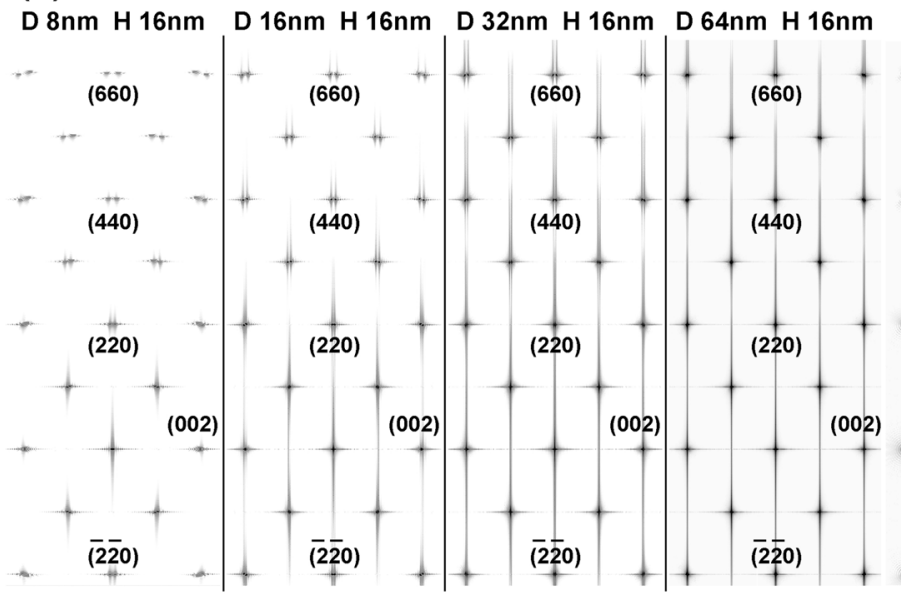

easily observed in the corresponding RS map (c) which also shows the split in two maxima of some reflections. This effect tends to decrease for increasing diameter, as the corresponding deviatoric strain also decreases with the radial distance. For the orientation used in (c) RS maps (vertical axis along [hh0]), the spread of intensity ("streaking") is caused by interference from cylinder (parallel) bases, an effect more pronounced for increasing diameter.

As an effect of the dislocation strain and twist, if the cylinder is long enough, upper/lower regions tend to lose correlation, as if the rod were made of different sub-domains. This is shown for the powder patterns in ((d) and (e)) and corresponding RS maps (f): here it is clearly visible that the $\{\mathrm{h} \bar{h} 0\}$ peaks, and all other peaks from atomic planes with the same [hh0] zone axis (all planes perpendicular to the cylinder circular cross-section) spread over an angular range. This angular dispersion increases with the distance from RS origin as a typical strain-related $\mathrm{effect}^{26}$ and is more pronounced for lower $\mathrm{D} / \mathrm{H}$ values. All of this is in good agreement with the Eshelby formula, as the total twist is proportional to $\mathrm{H} / \mathrm{D}^{2}$.

It is worth testing powder diffraction modelling procedures on DSE simulated data. Powder patterns from MD

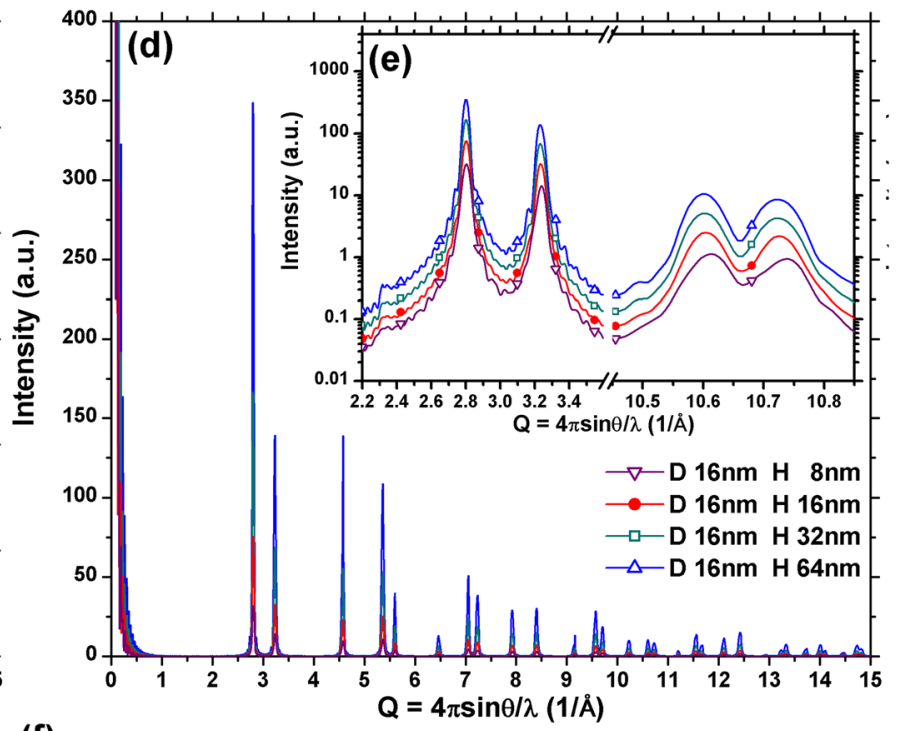

(f)

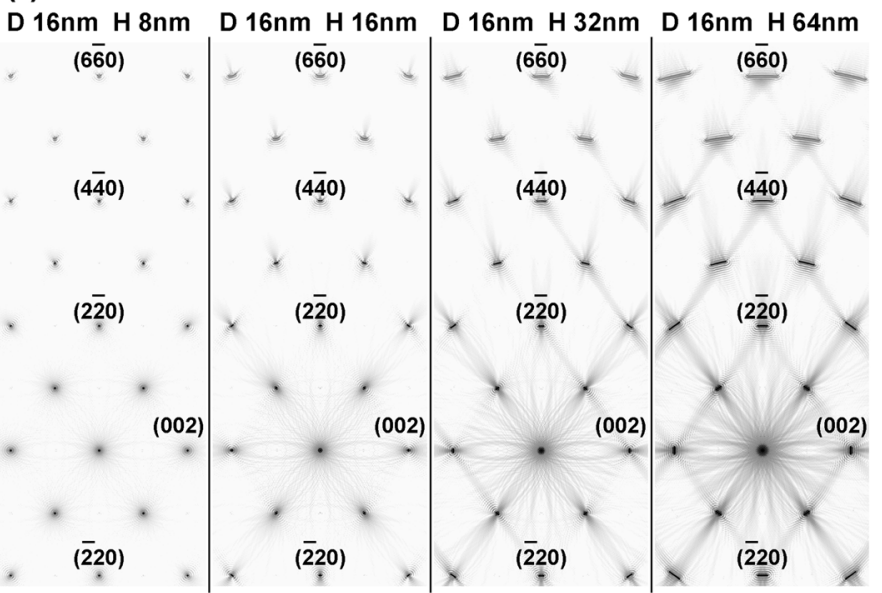

FIG. 8. Powder diffraction patterns from MD simulation of cylinders with screw dislocation along the [hh0] axis for constant height (a) and constant diameter (d). Details are shown in the insets, respectively, (b) and (e). Corresponding cross-sections of reciprocal space intensity distributions are shown below (respectively, in (c) and (f)). 
simulations can be used as experimental data and analyzed by a modern Line Profile Analysis, like Whole Powder Pattern Modelling (WPPM). ${ }^{27-29}$ Details on WPPM are easily found in the extensive published literature: in brief, diffraction line profiles across the whole available powder pattern are described by profile functions directly related to a microstructural model of the material; contributions of finite domain size and lattice defects are simultaneously accounted for by a convolution of the corresponding line profile components. Such a convolution, which supports a least squares minimization algorithm to fit experimental data (in the present case, DSE patterns from MD simulations), is carried out in terms of Fourier Transforms (FT) of the profile components arising from the different microstructural effects. No arbitrary adaptive profile functions are used, and the entire powder pattern can be modelled in terms of a few microstructural parameters. In the present case, the contribution of the finite cylindrical crystalline domains is represented by a corresponding analytical $\mathrm{FT}$ and allows refining $\mathrm{D}$ and $\mathrm{H},{ }^{30}$ whereas dislocation strain broadening is described by the FT expression given by the Krivoglaz-Wilkens theory. ${ }^{6-11}$ The latter involves the Burgers vector modulus (related to the $\mathrm{Pd}$ unit cell parameter, $a$, as $b=a / \sqrt{2}$ ), average dislocation density, $\rho$, effective outer cut-off radius, $R_{e}$, and average contrast factor, $\overline{\mathrm{C}}_{\mathrm{hkl}},{ }^{11,31,32}$ expressing the anisotropy of the dislocation strain field for the given slip system and the anisotropy of the elastic medium. ${ }^{11}$

According to the theory, the outer cut-off radius should be about twice the radius of Wilkens' restrictedly random dislocation region, ${ }^{10,11}$ which in this case reasonably coincides with the cylinder diameter $\mathrm{D}$, whereas the dislocation density, for a cylindrical domain, should theoretically be $4 /\left[\pi D^{2}\right]$, i.e., the inverse of the circular cross-section area.

Fig. 9 shows the WPPM result for the $\mathrm{D}=\mathrm{H}=16 \mathrm{~nm}$ cylinder without (a) and with (b) the screw dislocation along the [hh0] cylinder axis. The two powder patterns were modelled together, linking corresponding parameters. The result
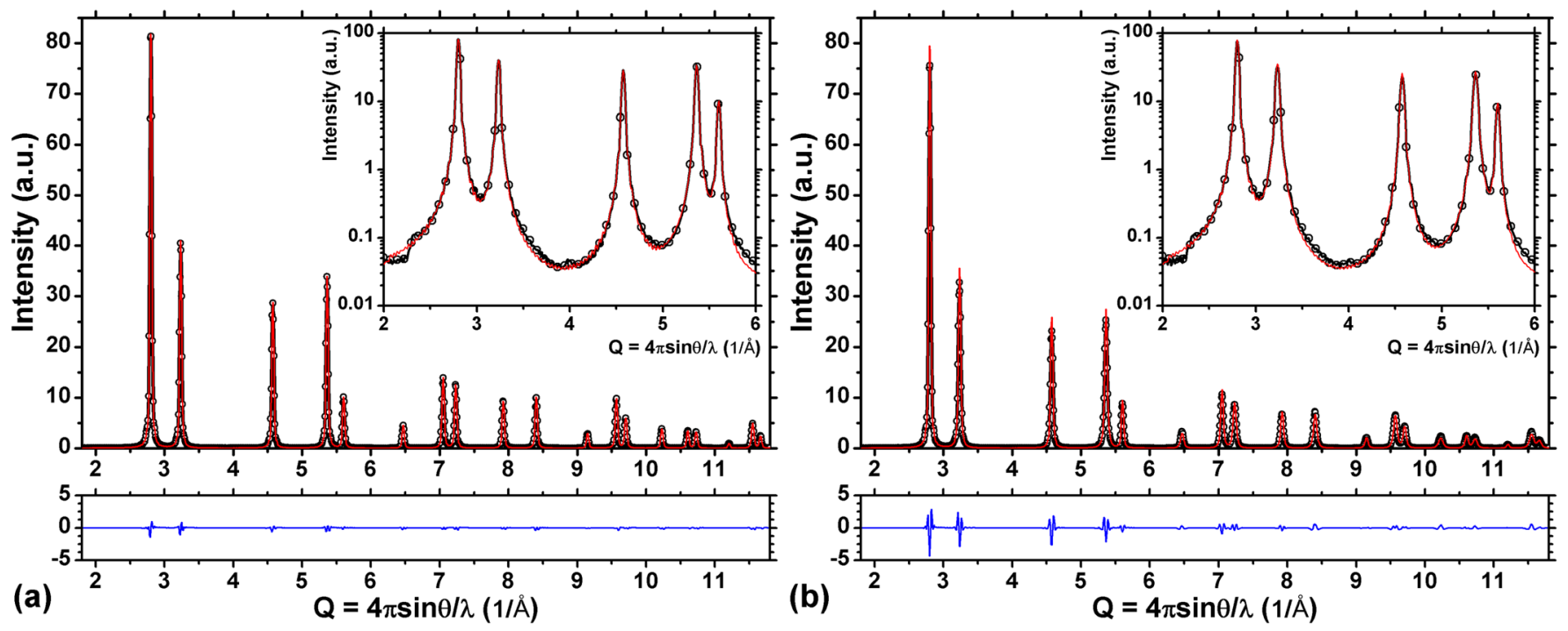

FIG. 9. WPPM results for the powder pattern of a $\mathrm{D}=\mathrm{H}=16 \mathrm{~nm}$ cylinder without (a) and with (b) a screw dislocation along the [hh0] cylinder axis. Details are shown in the insets. Data are obtained from MD simulations (circle), whereas the line is the least-squares modelling (WPPM) result. Line below (residual) is the difference between data and WPPM result. for the cylinder free of line defect is nearly perfect (flat residual), with refined cylinder size values in close agreement with the expected ones. It is indeed possible to fix $\mathrm{D}=\mathrm{H}=16 \mathrm{~nm}$ in the WPPM analysis with no significant effect on the modelling quality. The small but measurable strain caused by surface relaxation was modelled in terms of a microstrain function similar to that proposed by Adler and Houska, ${ }^{33,34}$ with the important additional consideration of strain anisotropy. The latter was based on a fourth order invariant function of the Miller indices first proposed by Popa. ${ }^{31,32,35}$

In the WPPM analysis of the cylinder with screw dislocation (Fig. 9(b)), the strain broadening component from surface relaxation was convolved with the dislocation strain broadening component. WPPM is not as perfect as in Fig. 9(a), a result visually demonstrated by the modelling residual. Refined parameters correspondingly differ from the expected values: results are shown in Fig. 10 for cylinders of different sizes, all with a dislocation along the [hh0] axis.

Refinements tend to be unstable when all parameters are simultaneously optimized. For this reason a strategy was adopted to limit free parameters. Best results (i.e., better agreement between refined parameters and nominal values of dislocation density, cut-off radius and cylinder (H and D) size) were obtained for cylinders with $\mathrm{D}=\mathrm{H}$ (open green symbols) which, as already pointed out, better conform to the restrictions of Pd cubic symmetry. However, refined dislocation density is always smaller than the nominal $\left(4 /\left[\pi D^{2}\right]\right)$ value, which implicitly assumes the strain field of an ideal straight dislocation. This is clearly the effect of the Eshelby twist, which partly relaxes the shear strain of the dislocation. Deviations are larger for $\mathrm{D} \neq \mathrm{H}$ cylinders, with the only significant exception of the $H=16 \mathrm{~nm}, \mathrm{D}=64 \mathrm{~nm}$ cylinder where the twist (proportional to $\mathrm{H} / \mathrm{D}^{2}$ ) is smallest, so the strain field is closer to a screw dislocation in an infinite medium (red full squares).

It is interesting to see the result for cylinders of different height and constant (fixed) $\mathrm{D}=16 \mathrm{~nm}$ (blue full squares). As is the difference between data and WPPM result. 

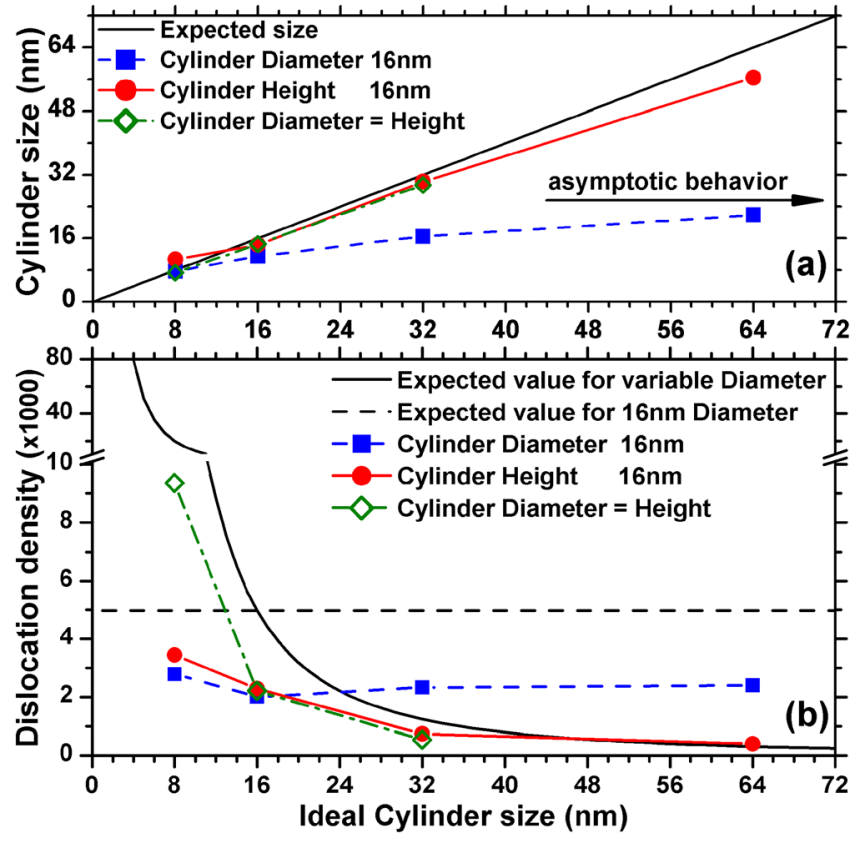

FIG. 10. WPPM results for cylinders of different sizes, all with a dislocation along the [hh0] axis. See text for details.

in previous cases the refined dislocation density is systematically smaller than (about half) the nominal value. In addition to that, for increasing $\mathrm{H}$, which in this set of simulations also means increasing H/D, refined heights increasingly deviate from the expected values. As in Figs. 8(d) and 8(e), this is caused by a larger than expected broadening, which is seen by the modelling as a smaller $\mathrm{H}$ effect. Same comments as those concerning Figs. 8(d), 8(e), and 8(f) hold here: in cylinders with high H/D (i.e., needle-like cylinders) the upper/lower regions of the crystalline domain tend to lose correlation because of the twist. The latter, for a constant diameter, linearly grows with the cylinder height, causing a progressive loss of correlation which is read by diffraction as a domain size effect: cylindrical nanocrystals appear as made of smaller, incoherently scattering sub-domains along the [hh0] axis.

To better understand this feature, besides referring to the RS map in Fig. 8(f), it is interesting to see RS maps and powder patterns for the same cylindrical rod with no dislocation. Cylinders with $\mathrm{D}=16 \mathrm{~nm}$ were made of two $\mathrm{H}=16 \mathrm{~nm}$ parts one on top of the other, with the upper one rotated by different angles around the common [hh0] axis (and no shift from the ideal structure spacing). These additional MD simulations mimic the twist effect in a sharper and more extreme way. Fig. 11 shows RS maps with reflections from atomic planes sharing the [hh0] (cylinder axis) zone axis (same orientation as in Fig. 8(f)): all points tend to split forming two clearly distinct maxima, a feature clearly visible for tilt angles larger than $\sim 0.5^{\circ}$. The analogy to the twist effect is quite evident: the spread of intensity over an angular range in Fig. 8(f), given by the progressive loss of correlation caused by the twist, here is replaced by the split of intensity maxima when the two $\mathrm{H}=16 \mathrm{~nm}$ cylinders are misaligned by more than $\sim 0.5^{\circ}$.

A WPPM analysis of the corresponding powder patterns shows (Fig. 11) that for tilt angles $>1.5^{\circ}$ coherence between
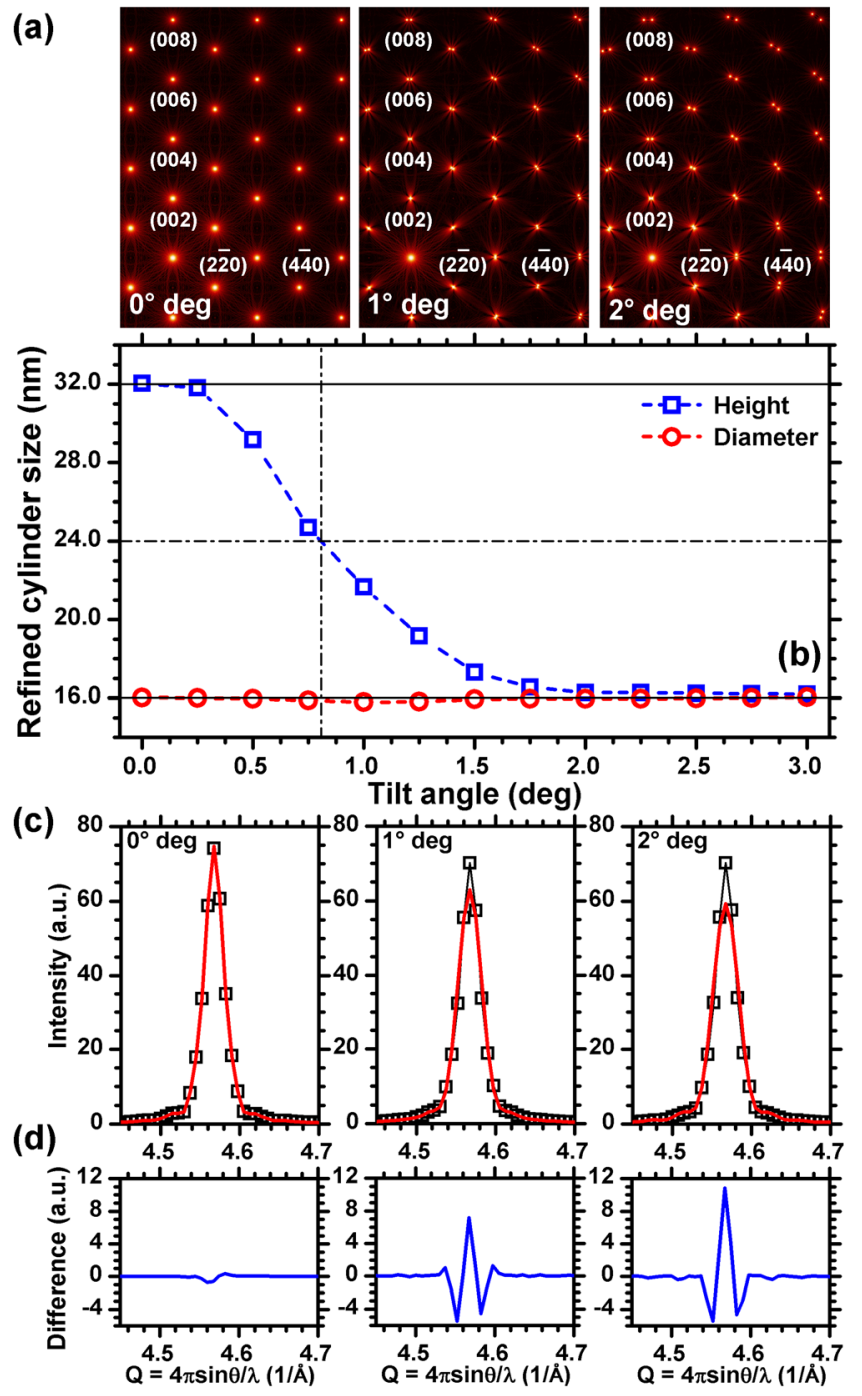

FIG. 11. Reciprocal space maps for $\mathrm{D}=16 \mathrm{~nm}$ cylinders made of two $\mathrm{H}=16 \mathrm{~nm}$ parts, rotated by different angles around the cylinder axis (a). WPPM results for D and $\mathrm{H}$ are shown in (b), whereas (c) shows a detail of the modelling in the angular region encompassing the (hh0) peak of cubic $\mathrm{Pd}$, and (d) the difference between simulated pattern and WPPM.

the two half-cylinders is completely lost, so that powder diffraction "sees" completely separate (incoherently scattering) domains of exactly $\mathrm{H}=16 \mathrm{~nm}$. Transition from $\mathrm{H}=32 \mathrm{~nm}$ single-crystal to two $\mathrm{H}=16 \mathrm{~nm}$ crystals is observed across a tilt angle range, between about $0.2^{\circ}$ and $1.5^{\circ}$, where WPPM provides intermediate $H$ values.

As a fine feature of these simulations, it is interesting to observe the evolution of the modelling quality for the (hh0) peak (Figs. 11(b)-11(d)). Data simulated by the DSE, being powder diffraction patterns from a cubic $(\mathrm{Pd})$ phase, include profile sub-components from all equivalent combinations of Miller indices; these are 12 for the $\{\mathrm{hh} 0\}$ family, two of which refer to the cylinder axis, [hh0], which is the only direction along which correlation is not influenced by the tilt. This is the reason why the modelling, which is nearly perfect with no tilt, gives a significant residual for large tilts (above $1.5^{\circ}$ ), when crystalline domains appear to be $\mathrm{D}=\mathrm{H}=16 \mathrm{~nm}$ for all other directions. In some way, this detail shows the global robustness of the WPPM approach, 
and of powder diffraction in general, as the final result is determined by the whole pattern, with contributions from many peaks and as many different crystallographic directions.

\section{CONCLUSIONS}

Dislocations in nanorods are significantly different from the straight line model frequently employed by continuum mechanics, but the solution proposed by Eshelby is in good agreement with the observed twist angle and deviatoric strain, even though it ignores surface stress and the splitting of dislocation into two partials.

In this work by using atomistic simulations we have proved that dislocations in nanorods produce effects that can be observed using either single-crystal or powder diffraction techniques. Thus laying the groundwork for experimental observation and data analysis. The most significant results we observed were:

(1) The Eshelby twist equation should be corrected to include the splitting of dislocations into partials, even if the effect on the twist angle is negligible, for diameters that are large compared to the distance between the partials;

(2) Twist angle values based on atomistic simulations analysis using a standard EAM approach are in good agreement with Eshelby continuum mechanics predictions, despite the differences between the ideal straight line screw dislocation assumed in the Eshelby model, and a more realistic atomic structure offered by $\mathrm{MD}$, where the dislocation line is split into partials and is itself twisted; moreover, the strain field is also partly affected by the free surfaces, because of the surface relaxation effect (lower coordination of surface atoms). Significant deviations from Eshelby formula are observed in disk-like cylinders, where the free surface constraint and strain are more relevant than in needle-like rods.

(3) Free surfaces and axial dislocation cause a distortion of the crystal lattice; this can be seen either in terms of distortion of the average cubic unit cell of Palladium or as deviatoric strain across the cylindrical nanocrystal. The latter significantly deviates from the traditional (continuum mechanics) expression for an ideal screw dislocation. Unlike the latter, strain in MD simulations (i) does not diverge in the core region, and (ii) does not decay monotonously with the distance; on the contrary, deviatoric strain increases again in the surface region because of the surface relaxation effect; in general, observed strain results from a combination of shear strain and twist effect, which MD simulations show to be (iii) always smaller than the strain predicted by the ideal continuum mechanics expressions (which refer to an ideal straight dislocation line in an infinite medium).

(4) All these features are visible in diffraction patterns, for both single crystals and powders. In agreement with the previous comments, an analysis of the powder pattern gives dislocation densities always below the nominal $\left(4 /\left[\pi D^{2}\right]\right)$ value. This is a further confirmation that the Eshelby twist partly relaxes the shear strain of an axial dislocation in a free rod. In addition to that, the twist weakens the correlation between more distant regions of the cylindrical domain, up to the point that needle-like nanocrystals appear as made of sub-domains (i.e., smaller sections along the cylinder axis) which scatter incoherently.

All these features should be relatively easy to observe in diffraction experiments on single-crystals or powders, thus providing a powerful tool for the study and practical applications of twisted nanocrystalline rods.

\section{ACKNOWLEDGMENTS}

The authors thank Ludovic Taxis for his careful review of the text and some useful suggestions. S.R. is supported by Basic Science Research Program through the National Research Foundation of Korea (NRF) funded by the Ministry of Science, ICT \& Future Planning (2013R1A1A010091). N.M.P. is supported by the European Research Council (ERC StG Ideas 2011 BIHSNAM no. 279985 on 'Bio-inspired hierarchical supernanomaterials', ERC PoC 2013-1 REPLICA2 no. 619448 on 'Large-area replication of biological anti-adhesive nanosurfaces', ERC PoC 2013-2 KNOTOUGH no. 632277 on 'Super-tough knotted fibres'), by the European Commission under the Graphene Flagship (WP10 'Nanocomposites', no. 604391) and by the Provincia Autonoma di Trento ('Graphene nanocomposites', no. S116/2012-242637 and reg. delib. no. 2266).

${ }^{1}$ J. D. Eshelby, J. Appl. Phys. 24, 176 (1953).

${ }^{2}$ J. Zhu, H. Peng, A. F. Marshall, D. M. Barnett, W. D. Nix, and Y. Cui, Nat. Nanotechnol. 3, 477 (2008).

${ }^{3}$ M. J. Bierman, Y. K. A. Lau, A. V. Kvit, A. L. Schmitt, and S. Jin, Science 320, 1060 (2008).

${ }^{4}$ F. Meng, S. A. Morin, A. Forticaux, and S. Jin, Acc. Chem. Res. 46, 1616 (2013).

${ }^{5}$ A. J. C. Wilson, Research 2, 541 (1949).

${ }^{6}$ M. A. Krivoglaz and K. P. Ryaboshapka, Fiz. Met. Metalloved. 15, 18 (1963) [Phys. Metals Metallogr. 15, 14-26 (1963)].

${ }^{7}$ M. A. Krivoglaz, O. V. Martynenko, and K. P. Ryaboshapka, Fiz. Met. Metalloved. 55, 5 (1983).

${ }^{8}$ R. I. Barabash and M. A. Krivoglaz, Sov. Sol. State Phys. 29, 3081 (1987). ${ }^{9}$ M. A. Krivoglaz, X-ray and Neutron Diffraction in Nonideal Crystals (Springer, 1996), p. 465.

${ }^{10} \mathrm{M}$. Wilkens, in Fundamental Aspects of Dislocation Theory, edited by J. A. Simmons, R. De Wit, and R. Bullough (Nat. Bur. Stand., US Spec. Publ. 317, II, Washington, DC, 1970), pp. 1195-1221.

${ }^{11}$ M. Wilkens, Phys. Status Solid A 2, 359 (1970).

${ }^{12}$ J. P. Hirth and J. Loathe, Theory of Dislocations (Wiley, New York, 1982).

${ }^{13}$ https://sites.google.com/site/eampotentials/Pd. See also: B. J. Lee, J. H. Shim, and M. Baskes, Phys. Rev. B 68, 144112 (2003); W. R. Tyson and W. A. Miller, Surf. Sci. 149, 407 (1977); G. Simons and H. Wang, Single Crystal Elastic Constants and Calculated Aggregate Properties (MIT Press, Cambridge, MA, 1977).

${ }^{14}$ R. O. Scattergood and D. J. Bacon, Philos. Mag. 31, 179 (1975).

${ }^{15}$ R. O. Scattergood and D. J. Bacon, Acta Metall. 30, 1665 (1982).

${ }^{16}$ C. R. Weinberger, Int. J. Plast. 27, 1391 (2011).

${ }^{17} \mathrm{D}$. Hull and D. J. Bacon, Introduction to Dislocations (ButterworthHeinemann, Oxford, 1965).

${ }^{18}$ S. Plimpton, J. Comput. Phys. 117, 1 (1995).

${ }^{19}$ M. S. Daw and M. I. Baskes, Phys. Rev. B 29, 6443 (1983).

${ }^{20}$ S. Foiles, M. Baskes, and M. S. Daw, Phys. Rev. B 33, 7983 (1986).

${ }^{21}$ H. W. Sheng, M. J. Kramer, A. Cadien, T. Fujita, and M. W. Chen, Phys. Rev. B 83, 134118 (2011). 
${ }^{22}$ A. Leonardi, M. Leoni, M. Li, and P. Scardi, J. Nanosci. Nanotechnol. 12, 8546 (2012).

${ }^{23}$ P. Debye, Annal. Phys. 351, 809 (1915).

${ }^{24}$ B. E. Warren, X-ray Diffraction (Dover, New York, 1990).

${ }^{25}$ L. Gelisio, C. L. Azanza Ricardo, M. Leoni, and P. Scardi, J. Appl. Cryst. 43, 647 (2010).

${ }^{26}$ L. Gelisio and P. Scardi, J. Nanosci. Nanotechnol. 12, 8811 (2012).

${ }^{27}$ P. Scardi and M. Leoni, Acta Cryst. A58, 190 (2002).

${ }^{28} \mathrm{P}$. Scardi and M. Leoni, in Diffraction Analysis of the Micro-Structure of Materials, edited by E. J. Mittemeijer and P. Scardi (Springer-Verlag, Berlin, Germany, 2004), pp. 51-91.
${ }^{29}$ P. Scardi, in Powder Diffraction: Theory and Practice, edited by R. E. Dinnebier and S. J. L. Billinge (The Royal Society of Chemistry, Cambridge, 2008), pp. 376-413.

${ }^{30}$ J. I. Langford and D. Louër, J. Appl. Cryst. 15, 20 (1982).

${ }^{31}$ M. Leoni, J. Martinez-Garcia, and P. Scardi, J. Appl. Cryst. 40, 719 (2007).

${ }^{32}$ J. Martinez-Garcia, M. Leoni, and P. Scardi, Acta Cryst. A 65, 109 (2009).

${ }^{33}$ T. Adler and C. R. Houska, J. Appl. Phys. 50, 3282 (1979).

${ }^{34}$ P. Scardi, A. Leonardi, L. Gelisio, M. R. Suchomel, B. T. Sneed, M. K. Sheehan, and C.-K. Tsung, Phys. Rev. B 91, 155414 (2015).

${ }^{35}$ N. C. Popa, J. Appl. Cryst. 31, 176 (1998). 